\title{
Large deviations for stochastic Volterra equations
}

\author{
DAVID NUALART* and CARLES ROVIRA
}

Facultat de Matemàtiques, Universitat de Barcelona, Gran Via 585, 08007 Barcelona, Spain. E-mail: *nualart@mat.ub.es

This paper is devoted to prove a large-deviation principle for solutions to multidimensional stochastic Volterra equations.

Keywords: large-deviations; stochastic Volterra equations

\section{Introduction}

The purpose of this paper is to study small perturbations of the solution to the stochastic Volterra equation

$$
X_{t}=x_{0}+\sum_{j=1}^{k} \int_{0}^{t} \sigma_{j}\left(t, s, X_{s}\right) \mathrm{d} W_{s}^{j}+\int_{0}^{t} b\left(t, s, X_{s}\right) \mathrm{d} s,
$$

$x_{0} \in \mathbb{R}^{d}, t \in[0, T]$. We will consider the family $\left\{X^{\varepsilon}, \varepsilon>0\right\}$ of processes which satisfy

$$
X_{t}^{\varepsilon}=x_{0}+\sum_{j=1}^{k} \int_{0}^{t} \varepsilon \sigma_{j}\left(t, s, X_{s}^{\varepsilon}\right) \mathrm{d} W_{s}^{j}+\int_{0}^{t} b\left(t, s, X_{s}^{\varepsilon}\right) \mathrm{d} s .
$$

We seek to establish a large-deviation principle (LDP) for $\left\{X^{\varepsilon}, \varepsilon>0\right\}$ on the space $\mathscr{C}_{x_{0}}\left([0, T], \mathbb{R}^{d}\right)$. We will prove the existence of a lower semi-continuous function $I: \mathscr{C}_{x_{0}}\left([0, T], \mathbb{R}^{d}\right) \rightarrow[0, \infty]$, called the rate function, such that $\{I \leqslant a\}$ is compact for any $a \in[0, \infty)$ and

$$
\begin{aligned}
& \lim _{\varepsilon \downarrow 0} \inf \varepsilon^{2} \log P\left\{X^{\varepsilon} \in O\right\} \geqslant-\Lambda(O), \\
& \lim _{\varepsilon \downarrow 0} \sup \varepsilon^{2} \log P\left\{X^{\varepsilon} \in F\right\} \leqslant-\Lambda(F),
\end{aligned}
$$

for any open set $O$ and closed set $F$ of $\mathscr{C}_{x_{0}}\left([0, T], \mathbb{R}^{d}\right)$, where, for a given subset $A \subset \mathscr{C}_{x_{0}}\left([0, T], \mathbb{R}^{d}\right), \Lambda(A)=\inf _{f \in A} I(f)$.

Large-deviation estimates were obtained by Ventzell and Freidlin (1970) for elliptic diffusions. Azencott (1980) and later Priouret (1982) extended the estimates to the general class of diffusions with Lipschitz coefficients. A principle of large-deviation uniformly with respect to the initial condition was proved in Millet et al. (1992). 
Stochastic Volterra equations in the plane are studied in Rovira and Sanz-Solé (1997), and an LDP is established assuming that the coefficients satisfy Lipschitz conditions in all the variables $t, s$ and $x$. Our aim is to prove an LDP for the solution of (1.1) assuming only Lipschitz continuity in $x$ and Hölder continuity in $t$ uniformly with respect to the other variables.

As an application of the LDP for stochastic Volterra equations we discuss in Section 5 two particular examples of interest: a stochastic differential equation driven by fractional Brownian motion and a hyperbolic stochastic partial differential equation. On the other hand, the study of stochastic Volterra equations provides a general framework that includes stochastic evolution equations.

The LDP is established using Azencott's method (Azencott 1980; Doss and Priouret $1983)$ in a general setting. More precisely, let $\left(\mathrm{E}_{i}, d_{i}\right), i=1,2$, be two Polish spaces and $X_{i}^{\varepsilon}: \Omega \rightarrow \mathrm{E}_{i}, \varepsilon>0, i=1,2$, families of random variables. Assume that $\left\{X_{1}^{\varepsilon}, \varepsilon>0\right\}$ satisfies an LDP with rate function $\tilde{I}: \mathrm{E}_{1} \rightarrow[0, \infty]$. Let $\Phi:\{\tilde{I}<+\infty\} \rightarrow E_{2}$ be a mapping such that its restriction to the compact sets $\{\tilde{I} \leqslant a\}, a \in[0, \infty)$, is continuous in the topology of $E_{1}$. For any $g \in E_{2}$ we set $I(g)=\inf \{\tilde{I}(f): \Phi(f)=g\}$. Suppose that for any $R, \rho, a>0$ there exist $\alpha>0$ and $\varepsilon_{0}>0$ such that for $f \in E_{1}$ satisfying $\tilde{I}(f) \leqslant a$ and $\varepsilon \leqslant \varepsilon_{0}$ we have

$$
P\left\{\mathrm{~d}_{2}\left(\mathrm{X}_{2}^{\varepsilon}, \Phi(f)\right) \geqslant \rho, d_{1}\left(X_{1}^{\varepsilon}, f\right)<\alpha\right\} \leqslant \exp \left(-\frac{R}{\varepsilon^{2}}\right) .
$$

Then, the family $\left\{X_{2}^{\varepsilon}, \varepsilon>0\right\}$ satisfies an LDP with rate function $I$.

Our aim is to apply this result to the random variables $X_{1}^{\varepsilon}=\varepsilon W$ and $X_{2}^{\varepsilon}=X^{\varepsilon}$ given in (1.2).

Although Azencott's method is well known, we have used two new ingredients in the proof of the LDP:

(i) We show an exponential inequality for stochastic integrals depending on a parameter. Due to the lack of the martingale property, the proof is based on the Garsia-RodemichRumsey lemma. Similar results have been obtained in Sowers (1992) and Rovira and SanzSolé (1996).

(ii) Unlike the case of diffusion processes, we are faced with stochastic integrals of the form

$$
\int_{t_{n}}^{t_{n+1}} \sigma\left(t, s, X_{t_{n}}\right) \mathrm{d} W_{s}
$$

which are not continuous functionals of $W$. For this reason, we need to use an LDP for the couple formed by $W$ and the above stochastic integral to obtain the suitable exponential bound. That is, we apply the LDP for Gaussian stochastic integrals depending on a parameter established in Ledoux (1990) and Mayer-Wolf et al. (1992).

The paper is organized as follows. In Section 2 we state the rigorous formulation of the problem and we present the main theorem, Theorem 1. The proof of this theorem requires an exponential inequality for stochastic integrals given in Section 3. In Section 4 we prove the LDP. Finally Section 5 contains two examples where we can apply this method. 
Sums on repeated indices are usually omitted and all constants are denoted by $C$, although they may change from one expression to the next.

\section{Formulation of the problem}

Let $\left(\Omega, \mathscr{F}, \mathscr{F}_{t}, P\right)$ be a stochastic basis carrying a $k$-dimensional $\mathscr{F}_{t}$-Brownian motion $\left\{W^{j}(t), 0 \leqslant t \leqslant T, 1 \leqslant j \leqslant k\right\}$. Let $x_{0} \in \mathbb{R}^{d}$ and consider the family $\left\{X^{\varepsilon}, \varepsilon>0\right\}$ of processes which satisfy (1.2).

To fomulate our results we assume the following set of assumptions $(\mathrm{H})$ :

$\left(\mathrm{H}_{1}\right)$ The functions $b=b(t, s, x), \sigma_{j}=\sigma_{j}(t, s, x), j=1, \ldots, k$, are measurable functions from $\{0 \leqslant s \leqslant t \leqslant T\} \times \mathbb{R}^{d}$ to $\mathbb{R}^{d}$.

$\left(\mathrm{H}_{2}\right)$ The functions $b, \sigma_{j}, j=1, \ldots, k$, are Lipschitz in $x$ (uniformly in the other variables). That is, there exists a constant $K$ such that

$$
\sum_{j=1}^{k}\left|\sigma_{j}(t, s, y)-\sigma_{j}(t, s, x)\right|+|b(t, s, y)-b(t, s, x)| \leqslant K|x-y|,
$$

for all $x, y \in \mathbb{R}^{d}, 0 \leqslant s \leqslant t \leqslant T$.

$\left(\mathrm{H}_{3}\right)$ The functions $b, \sigma_{j}, j=1, \ldots, k$, are $\alpha$-Hölder continuous in $t$ on $[s, T]$ (uniformly in the other variables). That is, there exists a constant $K$ such that

$$
\sum_{j=1}^{k}\left|\sigma_{j}(t, s, x)-\sigma_{j}(r, s, x)\right|+|b(t, s, x)-b(r, s, x)| \leqslant K|t-r|^{\alpha},
$$

for any $t, r \geqslant s$ and for all $x \in \mathbb{R}^{d}$, where $0<\alpha \leqslant 1$.

$\left(\mathrm{H}_{4}\right)$ There is a constant $K$ such that

$$
\sum_{j=1}^{k}\left|\sigma_{j}(t, s, x)-\sigma_{j}(r, s, x)-\sigma_{j}(t, s, y)+\sigma_{j}(r, s, y)\right| \leqslant K|t-r|^{\gamma}|x-y|,
$$

for any $t, r \geqslant s, x, y \in \mathbb{R}^{d}$, where $0<\gamma \leqslant 1$.

$\left(\mathrm{H}_{5}\right) b\left(t, s, x_{0}\right), \sigma_{j}\left(t, s, x_{0}\right), j=1, \ldots, k$, are bounded.

Let $\mathscr{H}$ be the Cameron-Martin space associated with $\mathscr{C}_{0}\left([0, T], \mathbb{R}^{k}\right)$, that is, the set of absolutely continuous functions $g \in \mathscr{C}_{0}\left([0, T], \mathbb{R}^{k}\right)$ with $\|g\|_{\mathscr{H}}^{2}:=\int_{0}^{T}\left|\dot{g}_{s}\right|^{2} \mathrm{~d} s<+\infty$, where $\dot{g}_{s}$ denotes the derivative $\mathrm{d} g / \mathrm{d} s$. Given $h \in \mathscr{H}$, we define the skeleton $S(h)$ by

$$
S(h)_{t}=x_{0}+\sum_{j=1}^{k} \int_{0}^{t} \sigma_{j}\left(t, s, S(h)_{s}\right) \dot{h}_{s}^{j} \mathrm{~d} s+\int_{0}^{t} b\left(t, s, S(h)_{s}\right) \mathrm{d} s .
$$

Our main result is as follows.

Theorem 1. Assume (H). The family $\left\{X^{\varepsilon}, \varepsilon \in(0,1]\right\}$ of solutions of (1.2) satisfies a largedeviation principle on the space $\mathscr{C}_{x_{0}}\left([0, T], \mathbb{R}^{d}\right)$ with rate function 


$$
I(\varphi)=\inf \left\{\frac{1}{2} \int_{0}^{T}\left|\dot{g}_{s}\right|^{2} \mathrm{~d} s, \varphi=S(g), g \in \mathscr{H}\right\},
$$

with $S(h)$ given in (2.1).

\section{Exponential inequality}

In this section we prove an exponential inequality for a type of stochastic integrals that are not martingales. Related exponential inequalities have been obtained in Mueller (1991), Sowers (1992) and Rovira and Sanz-Solé (1996).

Theorem 2. Let $Z:[0, T] \times[0, T] \times \Omega \rightarrow \mathbb{R}^{d} \times \mathbb{R}^{k} \quad$ be $\quad$ a $\quad \mathscr{B}([0, T]) \otimes \mathscr{B}([0, T]) \otimes \mathscr{F}$ measurable process satisfying the following conditions:

(i) $Z(t, s)=0$ if $s>t$.

(ii) $Z(t, s)$ is $\mathscr{F}_{s}$-adapted.

(iii) There exists a positive random variable $\xi$ and $\alpha \in(0,2]$ such that for all $t$, $r \in[0, T]$

$$
\int_{0}^{\min (r, t)}|Z(t, s)-Z(r, s)|^{2} \mathrm{~d} s \leqslant \xi|t-r|^{\alpha} .
$$

Then, for any $\beta, 0<\beta \leqslant \min (1, \alpha)$, there exist positive constants $K_{1}$ (depending only on $\beta$ ), $K_{2}$ and $K_{3}$, such that

$$
P\left\{\sup _{0 \leqslant t \leqslant T}\left|\int_{0}^{t} Z_{j}(t, s) \mathrm{d} W_{s}^{j}\right|>L,\|Z\|_{\infty} \leqslant K_{Z}, \xi \leqslant C_{Z}\right\} \leqslant \exp \left(-\frac{L^{2}}{\left(T K_{Z}^{2}+T^{\alpha} C_{Z}\right)} K_{3}\right)
$$

for any $L \geqslant 0, C_{Z} \geqslant 0$ and $K_{Z} \geqslant 0$ such that $L\left(T^{\alpha-\beta} C_{Z}+T^{1-\beta} K_{Z}^{2}\right)^{-1 / 2} \geqslant \max \left(K_{1}\right.$, $\left.K_{2}(1+T) T^{\beta / 2}\right)$.

Proof. We will prove the result in the case $k=d=1$. The extension to the general case is not difficult. Set $I(t):=\int_{0}^{t} Z(t, s) \mathrm{d} W_{s}$. For any $0 \leqslant r<t \leqslant T$ we have

$$
I(t)-I(r)=\int_{0}^{T} g_{t, r}(s) \mathrm{d} W_{s}
$$

with $g_{t, r}(s)=\rrbracket_{\{r<s \leqslant t\}} Z(t, s)+\mathfrak{q}_{\{s \leqslant r\}}(Z(t, s)-Z(r, s))$. Then

$$
\begin{aligned}
\int_{0}^{T} g_{t, r}^{2}(s) \mathrm{d} s & =\int_{r}^{t} Z^{2}(t, s) \mathrm{d} s+\int_{0}^{r}(Z(t, s)-Z(r, s))^{2} \mathrm{~d} s \\
& \leqslant|t-r|\|Z\|_{\infty}^{2}+|t-r|^{\alpha} \xi \\
& \leqslant|t-r|^{\beta}\left(T^{1-\beta}\|Z\|_{\infty}^{2}+T^{\alpha-\beta} \xi\right) .
\end{aligned}
$$


Set $\rho(y)=y^{\beta / 2} N_{\beta}^{1 / 2}, y \geqslant 0$ with $N_{\beta}:=T^{1-\beta} K_{Z}^{2}+T^{\alpha-\beta} C_{Z}$ and $\psi(x)=\exp \left(x^{2} / 4\right), x \in \mathbb{R}$. Let

$$
B=\int_{0}^{T} \int_{0}^{T} \psi\left(\frac{I(t)-I(r)}{\rho(|t-r|)}\right) \mathrm{d} t \mathrm{~d} r .
$$

For fixed $r<t$, we consider the continuous $\mathscr{F}_{u}$-martingale defined by

$$
M_{u}=\int_{0}^{u} \frac{g_{t, r}(s)}{\rho(|t-r|)} \mathrm{d} W_{s}
$$

with quadratic variation

$$
\langle M\rangle_{u} \leqslant \int_{0}^{T} \frac{g_{t, r}^{2}(s)}{\rho^{2}(|t-r|)} \mathrm{d} s \leqslant \frac{T^{1-\beta}\|Z\|_{\infty}^{2}+T^{\alpha-\beta} \xi}{N_{\beta}},
$$

for $0 \leqslant u \leqslant T$. Set $A=\left\{\|Z\|_{\infty} \leqslant K_{Z}, \xi \leqslant C_{Z}\right\}$. Note that on the set $A$ we have $\langle M\rangle_{T} \leqslant 1$. Then

$$
\mathrm{E}\left[\psi\left(\frac{I(t)-I(r)}{\rho(|t-r|)}\right) \rrbracket_{A}\right]=\mathrm{E}\left[\exp \left(\frac{M_{T}^{2}}{4}\right) \rrbracket_{A}\right] \leqslant \mathrm{E}\left[\exp \left\{\frac{1}{4}\left(\sup _{0 \leqslant r \leqslant 1}\left|Y_{r}\right|^{2}\right)\right\}\right]=\sqrt{2},
$$

where $Y$ is a Brownian motion such that $M_{u}=Y_{\langle M\rangle_{u}}$. So $\left.\mathrm{E}(B \rrbracket]_{A}\right) \leqslant T^{2} \sqrt{2}$ and

$$
\left.\left.\mathrm{E}\left(\exp \{\urcorner_{A}\left(\ln ^{+} B\right)\right\}\right) \leqslant 1+\mathrm{E}(\urcorner_{A} \exp \left(\ln ^{+} B\right)\right) \leqslant 2+T^{2} \sqrt{2},
$$

where $\ln +z=\max \{\ln z, 0\}$. Then, the Garsia-Rodemich-Rumsey lemma (Walsh 1986) yields, for any $\omega \in A$, and $t, r \in[0, T]$,

$$
|I(t)-I(r)| \leqslant 8 \int_{0}^{|t-r|} \psi^{-1}\left(\frac{B}{y^{2}}\right) \mathrm{d} \rho(y)
$$

and since $I(0)=0$,

$$
\|I\|_{\infty} \leqslant \sup _{0 \leqslant t \leqslant T} 8\left|\int_{0}^{t} \psi^{-1}\left(\frac{B}{y^{2}}\right) \mathrm{d} \rho(y)\right| \leqslant 2^{9 / 2}\left|\int_{0}^{T}\left(\left(\ln ^{+} B\right)^{1 / 2}+\left(\ln ^{+} y^{-2}\right)^{1 / 2}\right) \mathrm{d} \rho(y)\right| .
$$

Further calculations show that

$$
\int_{0}^{T}\left(\ln ^{+} y^{-2}\right)^{1 / 2} y^{\beta / 2-1} \mathrm{~d} y \leqslant 2 \sqrt{\pi} \beta^{-3 / 2} .
$$

Hence,

$$
\begin{aligned}
\|I\|_{\infty} & \leqslant 2^{9 / 2}\left[\left(\ln ^{+} B\right)^{1 / 2} \rho(T)+N_{\beta}^{1 / 2} \beta^{-1 / 2} \pi^{1 / 2}\right] \\
& =N_{\beta}^{1 / 2} C_{1}\left[T^{\beta / 2}\left(\ln ^{+} B\right)^{1 / 2}+C_{2}\right]
\end{aligned}
$$


with $C_{1}=2^{9 / 2}$ and $C_{2}=\beta^{-1 / 2} \pi^{1 / 2}$.

Now, from (3.1) and Chebyshev's exponential inequality, we obtain for $L / N_{\beta}^{1 / 2} \geqslant C_{1} C_{2}$,

$$
\begin{aligned}
P\left\{\left(\|I\|_{\infty} \geqslant L\right) \cap A\right\} & \leqslant P\left\{\left(\left(\ln ^{+} B\right) \geqslant\left(\frac{L}{C_{1} N_{\beta}^{1 / 2}}-C_{2}\right)^{2} \frac{1}{T^{\beta}}\right) \cap A\right\} \\
& \leqslant \operatorname{E}\left[\exp \left(\rrbracket_{A}\left(\ln ^{+} B\right)\right)\right] \exp \left\{-\left(\frac{L}{C_{1} N_{\beta}^{1 / 2}}-C_{2}\right)^{2} \frac{1}{T^{\beta}}\right\} \\
& \leqslant \exp \left\{-\left(\frac{L}{C_{1} N_{\beta}^{1 / 2}}-C_{2}\right)^{2} \frac{1}{T^{\beta}}+\ln \left(2+T^{2} \sqrt{2}\right)\right\} \\
& =\exp \left\{-\frac{L^{2}}{N_{\beta} T^{\beta}}\left[\left(\frac{1}{C_{1}}-\frac{C_{2} N_{\beta}^{1 / 2}}{L}\right)^{2}-\ln \left(2+T^{2} \sqrt{2}\right) \frac{N_{\beta} T^{\beta}}{L^{2}}\right]\right\} .
\end{aligned}
$$

Set $C_{3}=\max \left\{2 C_{1} C_{2}, 2^{3 / 2}\left(\ln \left(2+T^{2} \sqrt{2}\right)\right)^{1 / 2} C_{1} T^{\beta / 2}\right\}, C_{4}=1 /\left(8 C_{1}^{2}\right)$ and let $L / N_{\beta}^{1 / 2} \geqslant C_{3}$. Then,

$$
\begin{aligned}
P\left\{\left(\|I\|_{\infty} \geqslant L\right) \cap A\right\} & \leqslant \exp \left\{-\frac{L^{2}}{N_{\beta} T^{\beta}} 8 C_{4}\left[\left(1-\frac{N_{\beta}^{1 / 2}}{L} C_{2} C_{1}\right)^{2}-\frac{N_{\beta}}{L^{2}} \ln \left(2+T^{2} \sqrt{2}\right) C_{1}^{2} T^{\beta}\right]\right\} \\
& \leqslant \exp \left\{-\frac{L^{2}}{N_{\beta} T^{\beta}} C_{4}\right\} .
\end{aligned}
$$

Then it suffices to choose $K_{1}=2 C_{1} C_{2}=2^{11 / 2} \beta^{-1 / 2} \sqrt{\pi}, K_{2}=2^{25 / 4}$ and $K_{3}=C_{4}=2^{-12}$.

\section{Large deviations}

In this section we prove Theorem 1 . We will obtain the result by transferring a VentzellFreidlin type estimate, (see (1.3)) in Theorem 4. First, we need to study the continuity of the map $h \rightarrow S(h)$ defined in $(2.1)$.

Lemma 3. Under $(H)$ the map $h \rightarrow S(h)$ is continuous from $\left\{\|h\|_{\mathscr{H}} \leqslant a\right\}, a \in(0, \infty)$, into $\mathscr{C}_{x_{0}}\left([0, T], \mathbb{R}^{d}\right)$ with respect to the uniform norm.

Proof. Fix $g, h \in\left\{\|\cdot\|_{\mathscr{C}} \leqslant a\right\}$. Using the Lipschitz properties of $\sigma$ and $b$, we have 


$$
\left|S(g)_{t}-S(h)_{t}\right| \leqslant K \int_{0}^{t}\left|S(g)_{s}-S(h)_{s}\right|\left(1+\left|\dot{g}_{s}\right|\right) \mathrm{d} s+\left|\int_{0}^{t} \sigma_{j}\left(t, s, S(h)_{s}\right)\left(\dot{g}_{s}^{j}-\dot{h}_{s}^{j}\right) \mathrm{d} s\right|
$$

and, by Gronwall's lemma,

$$
\|S(g)-S(h)\|_{\infty} \leqslant C \sup _{0 \leqslant t \leqslant T}\left|\int_{0}^{t} \sigma_{j}\left(t, s, S(h)_{s}\right)\left(\dot{g}_{s}^{j}-\dot{h}_{s}^{j}\right) \mathrm{d} s\right|,
$$

for a constant $C>0$ depending on $a$.

Consider the function

$$
\rho(u)= \begin{cases}\exp \left\{-\bar{C} /\left(u^{2}-1\right)\right\} & \text { if }|u|<1, \\ 0, & \text { otherwise }\end{cases}
$$

and $\rho_{n}(u):=n \rho(n u)$, with $\bar{C}$ such that $\int_{-1}^{1} \rho(u) \mathrm{d} u=1$. By a standard procedure, if we put $\phi_{n}=\sigma * \rho_{n}$, that is,

$$
\phi_{n}(t, s)=\int_{0}^{t} \sigma\left(t, u, S(h)_{u}\right) \rho_{n}(s-u) \mathrm{d} u,
$$

then $s \rightarrow \phi_{n}(t, s)$ is continuously differentiable and $\phi_{n}(t,$.$) converges in L^{2}$ to $\sigma(t, ., S(h)$.). Moreover, from $\left(\mathrm{H}_{3}\right)$ it is easy to check that $t \rightarrow \phi_{n}(t, s)$ is $\alpha$-Hölder continuous in $t$ uniformly in $s$ (with constant $K_{n}$ ).

Fix $\varepsilon>0$. For any $\delta>0$, there exists a partition $0=t_{1}<t_{2}<\cdots<t_{N}=T$ such that $\sup _{i}\left|t_{i+1}-t_{i}\right| \leqslant \delta$ and $n_{0} \in N$ such that for any $n \geqslant n_{0}$

$$
\sup _{1 \leqslant i \leqslant N} \int_{0}^{T}\left|\sigma\left(t_{i}, s, S(h)_{s}\right)-\phi_{n}\left(t_{i}, s\right)\right|^{2} \mathrm{~d} s \leqslant \frac{\varepsilon}{6},
$$

where by convention $\sigma(t, s, x)=0$ if $t<s$. For any $t \in\left[t_{i}, t_{i+1}\right)$, we have

$$
\begin{aligned}
\int_{0}^{t}\left|\sigma\left(t, s, S(h)_{s}\right)-\phi_{n_{0}}(t, s)\right|^{2} \mathrm{~d} s & \\
\leqslant & 3\left[\int_{t_{i}}^{t}\left|\sigma\left(t, s, S(h)_{s}\right)\right|^{2} \mathrm{~d} s+\int_{0}^{t_{i}}\left|\sigma\left(t, s, S(h)_{s}\right)-\sigma\left(t_{i}, s, S(h)_{s}\right)\right|^{2} \mathrm{~d} s\right. \\
& \left.+\int_{0}^{t}\left|\sigma\left(t_{i}, s, S(h)_{s}\right)-\phi_{n_{0}}\left(t_{i}, s\right)\right|^{2} \mathrm{~d} s+\int_{0}^{t}\left|\phi_{n_{0}}\left(t_{i}, s\right)-\phi_{n_{0}}(t, s)\right|^{2} \mathrm{~d} s\right] .
\end{aligned}
$$

Then, using the $\alpha$-Hölder continuity properties, we obtain

$$
\begin{aligned}
& \sup _{t \in[0, T]} \int_{0}^{t}\left|\sigma\left(t, s, S(h)_{s}\right)-\phi_{n_{0}}(t, s)\right|^{2} \mathrm{~d} s \\
& \quad \leqslant 3\left(\varepsilon / 6+T\left(K+K_{n}\right) \delta^{2 \alpha}+\sup _{0 \leqslant s, t \leqslant T}\left|\sigma\left(t, s, S(h)_{s}\right)\right|^{2} \delta\right)=\varepsilon,
\end{aligned}
$$

if

$$
\delta=\min \left(\left(\frac{\varepsilon}{T\left(K+K_{n}\right) 6}\right)^{1 /(2 \alpha)}, \frac{\varepsilon}{\sup _{0 \leqslant s, t \leqslant T}\left|\sigma\left(t, s, S(h)_{s}\right)\right|^{2}}\right)
$$


On the other hand, notice that there exists a constant $C>0$ such that $\|S(h)\|_{\infty} \leqslant C$ and

$$
\sup _{0 \leqslant s, t \leqslant T}\left(\left|\phi_{n_{0}}(t, s)\right|+\left|\frac{\partial \phi_{n_{0}}}{\partial s}(t, s)\right|\right) \leqslant n_{0} C .
$$

Then, using Hölder's inequality and integration by parts,

$$
\begin{aligned}
\left|\int_{0}^{t} \sigma_{j}\left(t, s, S(h)_{s}\right)\left(\dot{g}_{s}^{j}-\dot{h}_{s}^{j}\right) \mathrm{d} s\right| \leqslant & \left|\int_{0}^{t}\left(\sigma_{j}\left(t, s, S(h)_{s}\right)-\phi_{n_{0}}^{j}(t, s)\right)\left(\dot{g}_{s}^{j}-\dot{h}_{s}^{j}\right) \mathrm{d} s\right| \\
& +\left|\int_{0}^{t} \phi_{n_{0}}^{j}(t, s)\left(\dot{g}_{s}^{j}-\dot{h}_{s}^{j}\right) \mathrm{d} s\right| \\
\leqslant & 2 a \varepsilon^{1 / 2}+\|h-g\|_{\infty}\left(\left\|\phi_{n_{0}}^{j}\right\|_{\infty}+\int_{0}^{T}\left|\frac{\partial \phi_{n_{0}}}{\partial s}(t, s)\right| \mathrm{d} s\right)
\end{aligned}
$$

and from (4.1) the proof is finished.

The main theorem is the following.

Theorem 4. Assume (H). For any $g \in \mathscr{H}, R, \rho>0$, there exist $\eta>0$ and $\varepsilon_{0}>0$ such that

$$
P\left\{\left\|X^{\varepsilon}-S(g)\right\|_{\infty}>\rho,\|\varepsilon W-g\|_{\infty}<\eta\right\} \leqslant \exp \left(-\frac{R}{\varepsilon^{2}}\right),
$$

for any $\varepsilon \in\left(0, \varepsilon_{0}\right]$.

Let $\left\{Y_{t}^{\varepsilon}, 0 \leqslant t \leqslant T\right\}$ be the solution process of

$$
Y_{t}^{\varepsilon}=x_{0}+\int_{0}^{t} \varepsilon \sigma_{j}\left(t, s, Y_{s}^{\varepsilon}\right) \mathrm{d} W_{s}^{j}+\int_{0}^{t} b\left(t, s, Y_{s}^{\varepsilon}\right) \mathrm{d} s+\int_{0}^{t} \sigma_{j}\left(t, s, Y_{s}^{\varepsilon}\right) \dot{g}_{s}^{j} \mathrm{~d} s .
$$

A reduced version of the proof of Theorem 4 can be used to establish the following result.

Proposition 5. Assume (H). For all $g \in \mathscr{H}_{0}, R, \rho>0$, there exist $\eta>0$ and $\varepsilon_{0} \in(0,1]$ such that

$$
P\left\{\left\|Y^{\varepsilon}-S(g)\right\|_{\infty}>\rho,\|\varepsilon W\|_{\infty}<\eta\right\} \leqslant \exp \left(-\frac{R}{\varepsilon^{2}}\right),
$$

for any $\varepsilon \in\left(0, \varepsilon_{0}\right]$.

Indeed, let $\bar{W}_{t}^{\varepsilon}=W_{t}-(1 / \varepsilon) g(t)$. Girsanov's theorem ensures that $\left\{\bar{W}_{t}^{\varepsilon}, 0 \leqslant t \leqslant T\right\}$ is a $k$-dimensional Wiener process with respect to the probability $P^{\varepsilon}$ given by

$$
\frac{\mathrm{d} P^{\varepsilon}}{\mathrm{d} P}=\exp \left\{\frac{1}{\varepsilon} \int_{0}^{T} \dot{g}^{j}(s) \mathrm{d} W_{s}^{j}-\frac{1}{2 \varepsilon^{2}} \int_{0}^{T}\left|\dot{g}_{s}\right|^{2} \mathrm{~d} s\right\} .
$$


Set, for any $\rho, \eta, \varepsilon>0$,

$$
\begin{aligned}
B^{\varepsilon} & =\left\{\left\|X^{\varepsilon}-S(g)\right\|_{\infty}>\rho,\|\varepsilon W-g\|_{\infty}<\eta\right\}, \\
U^{\varepsilon} & =\exp \left\{-\frac{1}{\varepsilon} \int_{0}^{T} \dot{g}^{j}(s) \mathrm{d} W_{s}^{j}\right\} .
\end{aligned}
$$

Then

$$
\begin{aligned}
P\left(B^{\varepsilon}\right) & \leqslant P\left\{B^{\varepsilon} \cap\left(U^{\varepsilon} \leqslant \exp \left(\frac{\lambda}{\varepsilon^{2}}\right)\right)\right\}+\mathrm{P}\left\{U^{\varepsilon}>\exp \left(\frac{\lambda}{\varepsilon^{2}}\right)\right\} \\
& \leqslant \exp \left(\frac{\lambda+a / 2}{\varepsilon^{2}}\right) P^{\varepsilon}\left(B^{\varepsilon}\right)+P\left\{-\frac{1}{\varepsilon} \int_{0}^{T} \dot{g}_{s}^{j} \mathrm{~d} W_{s}^{j}>\frac{\lambda}{\varepsilon^{2}}\right\},
\end{aligned}
$$

where $a=\|g\|_{\mathscr{H}}^{2}$ and $\lambda \in \mathbb{R}$. For $\lambda$ big enough, a well-known inequality for Gaussian random variables yields

$$
P\left\{\left|\int_{0}^{T} \dot{g}_{s}^{j} \mathrm{~d} W_{s}^{j}\right|>\frac{\lambda}{\varepsilon}\right\} \leqslant 2 \exp \left(-\frac{\lambda^{2}}{2 \varepsilon^{2} a}\right) \leqslant \exp \left(-\frac{R}{\varepsilon^{2}}\right) .
$$

Set $Y^{\varepsilon}\left(\bar{\omega}^{\varepsilon}\right)=X^{\varepsilon}\left(\bar{\omega}^{\varepsilon}+\frac{1}{\varepsilon} g\right)$. Then,

$$
P^{\varepsilon}\left(B^{\varepsilon}\right)=P\left\{\left\|Y^{\varepsilon}-S(g)\right\|_{\infty}>\rho,\|\varepsilon W\|_{\infty}<\eta\right\},
$$

with $Y^{\varepsilon}$ satisfying (4.2). Consequently, the estimates (4.4), (4.5) and Proposition 5 complete the proof of Theorem 4.

We first prove Proposition 5 under some assumptions stronger than $(\mathrm{H})$. We assume that the coefficients $\sigma$ and $b$ satisfy $(\mathrm{H})$ and, moreover, that they satisfy hypothesis $\left(\mathrm{H}_{+}\right)$, that is:

$\left(\mathrm{H}_{+}\right)$There exists a constant $\bar{K}>0$ such that

$$
\begin{aligned}
& \sigma(t, s, x)=\sigma\left(t, s, \frac{x}{|x|} \bar{K}\right), \\
& b(t, s, x)=b\left(t, s, \frac{x}{|x|} \bar{K}\right),
\end{aligned}
$$

for any $x,|x|>\bar{K}$ and for all $s \leqslant t$.

Notice that under such assumptions $\sigma$ and $b$ are bounded. We denote by $K$ a bound of these coefficients. The proof of Proposition 5 relies on the following lemma and propositions.

Lemma 6. Fix $g \in \mathscr{H},\|g\|_{\mathscr{C}} \leqslant$ a. There exists a non-negative constant $\bar{K}_{1}$ depending only on the coefficients and a such that P-a.s.

$$
\left\|Y^{\varepsilon}-S(g)\right\|_{\infty} \leqslant \bar{K}_{1} \sup _{0 \leqslant t \leqslant T}\left|\int_{0}^{t} \varepsilon \sigma_{j}\left(t, s, Y_{s}^{\varepsilon}\right) \mathrm{d} W_{s}^{j}\right| .
$$


Proof. From identities (4.2) and (2.1) and Gronwall's lemma it is easy to obtain the estimate (4.6).

We now consider a discretization of $Y^{\varepsilon}$. To this end we first introduce some notation. For $n \in \mathbb{N}, \quad l=0,1, \ldots, n$, set $t_{l}^{n}=T l /^{n}, \Delta_{l}^{n}=\left[t_{l}^{n}, t_{l+1}^{n}\right)$. Let $Y_{t}^{\varepsilon, n}=Y_{t_{l}^{n}}^{\varepsilon}$ and $t^{n}=t_{l}^{n}$ if $t \in \Delta_{l}^{n}$.

Proposition 7. For all $R>0$ and $\mu>0$ there exists $n_{0} \in \mathbb{N}$ (depending on $R$ and $\mu$ ), such that for all $n \geqslant n_{0}$ and $\varepsilon(0,1]$

$$
P\left\{\left\|Y^{\varepsilon}-Y^{\varepsilon, n}\right\|_{\infty}>\mu\right\} \leqslant \exp \left(-\frac{R}{\varepsilon^{2}}\right) .
$$

Proof. Fix $n$. For $t \in \Delta_{i}^{n}$, using condition $\left(H_{3}\right)$ and the boundedness of coefficients $\sigma$ and $b$, we have

$$
\begin{aligned}
\left|Y_{t}^{\varepsilon}-Y_{t}^{\varepsilon, n}\right| \leqslant & \left|Y_{t}^{\varepsilon}-Y_{t_{i}^{n}}^{\varepsilon}\right| \\
\leqslant & \varepsilon\left|\int_{t_{i}^{n}}^{t} \sigma_{j}\left(t, s, Y_{s}^{\varepsilon}\right) \mathrm{d} W_{s}^{j}\right|+\varepsilon\left|\int_{0}^{t_{i}^{n}}\left(\sigma_{j}\left(t, s, Y_{s}^{\varepsilon}\right)-\sigma_{j}\left(t_{i}^{n}, s, Y_{s}^{\varepsilon}\right)\right) \mathrm{d} W_{s}^{j}\right| \\
& +\mid \int_{0}^{t} b\left(t, s, Y_{s}^{\varepsilon}\right) \mathrm{d} s+\int_{0}^{t} \sigma_{j}\left(t, s, Y_{s}^{\varepsilon}\right) \dot{g}_{s}^{j} \mathrm{~d} s \\
& \quad-\int_{0}^{t_{i}^{n}} b\left(t_{i}^{n}, s, Y_{s}^{\varepsilon}\right) \mathrm{d} s-\int_{0}^{t_{i}^{n}} \sigma_{j}\left(t_{i}^{n}, s, Y_{s}^{\varepsilon}\right) \dot{g}_{s}^{j} \mathrm{~d} s \mid \\
\leqslant & \varepsilon\left|\int_{t_{i}^{n}}^{t} \sigma_{j}\left(t, s, Y_{s}^{\varepsilon}\right) \mathrm{d} W_{s}^{j}\right|+\varepsilon\left|\int_{0}^{t_{i}^{n}}\left(\sigma_{j}\left(t, s, Y_{s}^{\varepsilon}\right)-\sigma_{j}\left(t_{i}^{n}, s, Y_{s}^{\varepsilon}\right)\right) \mathrm{d} W_{s}^{j}\right| \\
& +K\left(a\left|t-t_{i}^{n}\right|^{1 / 2}+\left(T+a T^{1 / 2}\right)\left|t-t_{i}^{n}\right|^{\alpha}+\left|t-t_{i}^{n}\right|\right) .
\end{aligned}
$$

Then

$$
\begin{aligned}
P\left(\left\|Y^{\varepsilon}-Y^{\varepsilon, n}\right\|_{\infty}>\mu\right) \leqslant & \sum_{i=1}^{n}\left[P\left(\sup _{t \in \Delta_{i}^{n}}\left|\int_{t_{i}^{n}}^{t} \varepsilon \sigma_{j}\left(t, s, Y_{s}^{\varepsilon}\right) \mathrm{d} W_{s}^{j}\right|>\frac{\mu}{3}\right)\right. \\
& +P\left(\sup _{t \in \Delta_{i}^{n}} \varepsilon\left|\int_{0}^{t_{i}^{n}}\left(\sigma_{j}\left(t, s, Y_{s}^{\varepsilon}\right)-\sigma_{j}\left(t_{i}^{n}, s, Y_{s}^{\varepsilon}\right)\right) \mathrm{d} W_{s}^{j}\right|>\frac{\mu}{3}\right) \\
& \left.+P\left(\sup _{t \in \Delta_{i}^{n}} K\left(a\left|t-t_{i}^{n}\right|^{1 / 2}+\left(T+a T^{1 / 2}\right)\left|t-t_{i}^{n}\right|^{\alpha}+\left|t-t_{i}^{n}\right|\right)>\frac{\mu}{3}\right)\right] \\
= & \sum_{i=1}^{n}\left[P\left(A_{1}^{i}\right)+P\left(A_{2}^{i}\right)+P\left(A_{3}^{i}\right)\right] .
\end{aligned}
$$


Let $\beta<\min (1,2 \alpha)$. Theorem 2 ensures the existence of positive constants $K_{1}, K_{2}$ and $K_{3}$ such that

$$
P\left(A_{1}^{i}\right) \leqslant \exp \left(-\frac{\mu^{2}}{\varepsilon^{2} 9 K^{2}\left(\frac{T}{n}+\left(\frac{T}{n}\right)^{2 \alpha}\right)} K_{3}\right)
$$

whenever

$$
\mu /\left\{\varepsilon 3 K\left(\left(\frac{T}{n}\right)^{1-\beta}+\left(\frac{T}{n}\right)^{2 \alpha-\beta}\right)^{1 / 2}\right\} \geqslant \max (K)_{1}, K_{2}\left(1+\frac{T}{n}\right)\left(\frac{T}{n}\right)^{\beta / 2} .
$$

Now choose $\beta<\min (1, \alpha)$. Using hypothesis $\left(\mathrm{H}_{3}\right)$, Theorem 2 with $C_{Z}=K\left(\frac{T}{n}\right)^{\alpha}$ yields the existence of positive constants $K_{1}, K_{2}$ and $K_{3}$ such that

$$
P\left(A_{2}^{i}\right) \leqslant \exp \left(-\frac{\mu^{2}}{\varepsilon^{2} 9 K^{2}\left(T\left(\frac{T}{n}\right)^{2 \alpha}+T^{\alpha}\left(\frac{T}{n}\right)^{\alpha}\right)} K_{3}\right),
$$

whenever

$$
\mu /\left\{\varepsilon 3 K\left(T^{1-\beta}\left(\frac{T}{n}\right)^{2 \alpha}+T^{\alpha-\beta}\left(\frac{T}{n}\right)^{\alpha}\right)^{1 / 2}\right\} \geqslant \max \left(K_{1}, K_{2}(1+T) T^{\beta / 2} .\right.
$$

On the other hand, for $n$ big enough

$$
P\left(A_{3}^{i}\right)=0 .
$$

Finally, from (4.7)-(4.10) for $n$ big enough we obtain the desired result.

Proposition 8. For all $R>0, \rho>0, n \in \mathbb{N}$ there exist $\mu_{0}$ (depending only on $R$ and $\rho$ ) and $\eta_{0}>0$ (depending on $R, \rho$ and $n$ ) such that, for all $\mu \leqslant \mu_{0}, \eta \leqslant \eta_{0}$ and $\varepsilon \in(0,1]$,

$$
P\left\{\sup _{0 \leqslant t \leqslant T}\left|\int_{0}^{t} \varepsilon \sigma_{j}\left(t, s, Y_{s}^{\varepsilon}\right) \mathrm{d} W_{s}^{j}\right|>\rho,\|\varepsilon W\|_{\infty}<\eta,\left\|Y^{\varepsilon}-Y^{\varepsilon, n}\right\|_{\infty} \leqslant \mu\right\} \leqslant \exp \left(-\frac{R}{\varepsilon^{2}}\right) .
$$

Proof. We can write

$$
P\left\{\sup _{0 \leqslant t \leqslant T}\left|\int_{0}^{t} \varepsilon \sigma_{j}\left(t, s, Y_{s}^{\varepsilon}\right) \mathrm{d} W_{s}^{j}\right|>\rho,\|\varepsilon W\|_{\infty}<\eta,\left\|Y^{\varepsilon}-Y^{\varepsilon, n}\right\|_{\infty} \leqslant \mu\right\} \leqslant P_{1}+P_{2},
$$

with

$$
\begin{aligned}
& P_{1}:=P\left\{\sup _{0 \leqslant t \leqslant T}\left|\int_{0}^{t} \varepsilon\left(\sigma_{j}\left(t, s, Y_{s}^{\varepsilon}\right)-\sigma_{j}\left(t, s, Y_{s}^{\varepsilon, n}\right)\right) \mathrm{d} W_{s}^{j}\right|>\frac{\rho}{2},\left\|Y^{\varepsilon}-Y^{\varepsilon, n}\right\|_{\infty} \leqslant \mu\right\} \\
& P_{2}:=P\left\{\sup _{0 \leqslant t \leqslant T}\left|\int_{0}^{t} \varepsilon \sigma_{j}\left(t, s, Y_{s}^{\varepsilon, n}\right) \mathrm{d} W_{s}^{j}\right|>\frac{\rho}{2},\|\varepsilon W\|_{\infty}<\eta\right\} .
\end{aligned}
$$

Consider $Z(t, s)=\sigma\left(t, s, Y_{s}^{\varepsilon}\right)-\sigma\left(t, s, Y_{s}^{\varepsilon, n}\right)$. Then $\|Z\|_{\infty} \leqslant K\left\|Y_{s}^{\varepsilon}-Y_{s}^{\varepsilon, n}\right\|_{\infty}$ and from $\left(\mathrm{H}_{4}\right)$ we have, for any $t, r \geqslant s$, 


$$
|Z(t, s)-Z(r, s)|^{2} \leqslant K^{2}\left\|Y_{s}^{\varepsilon}-Y_{s}^{\varepsilon, n}\right\|_{\infty}^{2}|t-r|^{2 \gamma} .
$$

Theorem 2 yields the existence of positive constants $K_{1}, K_{2}$ and $K_{3}$ such that

$$
P_{1} \leqslant \exp \left(-\frac{\rho^{2}}{\varepsilon^{2} 4 K^{2}\left(T \mu^{2}+T^{2 \gamma+1} \mu^{2}\right)} K_{3}\right)
$$

if $\rho /\left\{\varepsilon 2 K\left(T^{1-\beta} \mu^{2}+T^{2 \gamma+1-\beta} \mu^{2}\right)^{1 / 2}\right\} \geqslant \max \left(K_{1}, K_{2}(1+T) T^{\beta / 2}\right)$, with $\beta=\min (1,2 \gamma)$. This is possible by choosing $\mu$ in a suitable way. On the other hand,

$$
\begin{aligned}
P_{2} & =P\left\{\sup _{0 \leqslant t \leqslant T}\left|\varepsilon \sum_{i=1}^{n} \int_{[0, t] \cap \Delta_{i}^{n}} \sigma_{j}\left(t, s, Y_{t_{i}^{n}}^{\varepsilon}\right) \mathrm{d} W_{s}^{j}\right|>\frac{\rho}{2},\|\varepsilon W\|_{\infty}<\eta\right\} \\
& \leqslant \sum_{i=1}^{n} P\left\{\sup _{0 \leqslant t \leqslant T}\left|\varepsilon \int_{[0, t] \cap \Delta_{i}^{n}} \sigma_{j}\left(t, s, Y_{t_{i}^{n}}^{\varepsilon}\right) \mathrm{d} W_{s}^{j}\right|>\frac{\rho}{2 n},\|\varepsilon W\|_{\infty}<\eta\right\} \\
& =\sum_{i=1}^{n} P\left(B_{i}\right) .
\end{aligned}
$$

Fix $i \in\{1, \ldots, n\}$ and define $\bar{W}_{t}=W_{t}-W_{t_{i}^{n}}$, for $t \geqslant t_{i}^{n}$. Then

$$
\int_{[0, t] \cap \Delta_{i}^{n}} \sigma_{j}\left(t, s, Y_{t_{i}^{n}}^{\varepsilon}\right) \mathrm{d} W_{s}^{j}=\int_{[0, t] \cap \Delta_{i}^{n}} \sigma_{j}\left(t, s, Y_{t_{i}^{n}}^{\varepsilon}\right) \mathrm{d} \bar{W}_{s}^{j} .
$$

Notice that $Y_{t_{i}^{n}}^{\varepsilon}$ is independent of $\left\{\bar{W}_{t}, t \geqslant t_{i}^{n}\right\}$. So

$$
\int_{[0, t] \cap \Delta_{i}^{n}} \sigma_{j}\left(t, s, Y_{t_{i}^{n}}^{\varepsilon}\right) \mathrm{d} \bar{W}_{s}^{j}=\left.\int_{[0, t] \cap \Delta_{i}^{n}} \sigma_{j}(t, s, y) \mathrm{d} \bar{W}_{s}^{j}\right|_{y=Y_{t_{i}^{n}}^{\varepsilon}}
$$

Using again the independence of $Y_{t_{i}^{n}}^{\varepsilon}$ and $\left\{\bar{W}_{t}, t \geqslant t_{i}^{n}\right\}$, we have

$$
\begin{aligned}
P\left(B_{i}\right) & \leqslant P\left(\sup _{0 \leqslant t \leqslant T}\left|\varepsilon \int_{[0, t] \cap \Delta_{i}^{n}} \sigma_{j}\left(t, s, Y_{t_{i}^{n}}^{\varepsilon}\right) \mathrm{d} W_{s}^{j}\right|>\frac{\rho}{2 n}, \sup _{t \geqslant t_{i}^{n}}\left|\varepsilon \bar{W}_{t}\right|<2 \eta\right) \\
& =\int_{\mathbb{R}} \mathrm{d} \mathscr{C}_{Y_{t_{i}^{\varepsilon}}^{\varepsilon}}(y) P\left\{\sup _{0 \leqslant t \leqslant T}\left|\varepsilon \int_{[0, t] \cap \Delta_{i}^{n}} \sigma_{j}(t, s, y) \mathrm{d} \bar{W}_{s}^{j}\right|>\frac{\rho}{2 n}, \sup _{t \geqslant t_{i}^{n}}\left|\varepsilon \bar{W}_{t}\right|<2 \eta\right\} .
\end{aligned}
$$

The Gaussian process $\left\{\left(\int_{[0, t] \cap \Delta_{i}^{n}} \sigma_{j}(t, s, y) \mathrm{d} \bar{W}_{s}^{j}, \bar{W}_{t}\right), t_{i} \leqslant t \leqslant T\right\}$ satisfies an LDP in $\mathscr{C}_{0}\left(\left[t_{i}, T\right], \mathbb{R}^{d} \otimes \mathbb{R}^{k}\right)$ with rate function

$$
I(h)=\inf \left\{\frac{1}{2} \int_{t_{i}}^{T}\left|\dot{g}_{s}\right|^{2} \mathrm{~d} s, h_{t}=\left(\int_{[0, t] \cap \Delta_{i}^{n}} \sigma_{j}(t, s, y) \dot{g}_{s}^{j} \mathrm{~d} s, g_{t}\right)\right\}
$$

(see, for instance, Mayer-Wolf et al. 1992; Ledoux 1990). Then, from the LDP, we have 


$$
\begin{aligned}
& P\left\{\sup _{0 \leqslant t \leqslant T}\left|\varepsilon \int_{[0, t] \cap \Delta_{i}^{n}} \sigma_{j}(t, s, y) \mathrm{d} \bar{W}_{s}^{j}\right|>\frac{\rho}{2 n}, \sup _{t \geqslant t_{i}^{n}}\left|\varepsilon \bar{W}_{t}\right|<2 \eta\right\} \\
& \leqslant \exp \left(-\frac{1}{\varepsilon^{2}} \inf \left\{\frac{1}{2} \int_{t_{i}}^{T}\left|\dot{g}_{s}\right|^{2} \mathrm{~d} s,\|g\|_{\infty} \leqslant 2 \eta, \sup _{t_{i} \leqslant t \leqslant T}\left|\int_{[0, t] \cap \Delta_{i}^{n}} \sigma_{j}(t, s, y) \dot{g}_{s}^{j} \mathrm{~d} s\right|>\frac{\rho}{2 n}\right\}\right) .
\end{aligned}
$$

Using the same arguments as in Lemma 3, it is easy to check that for each $R$, the map $g \rightarrow \int[0, t] \cap \Delta_{i}^{n} \sigma_{j}(t, s, y) \dot{g}_{s}^{j} \mathrm{~d} s$ is continuous in the uniform norm in $\left\{g, \frac{1}{2}\|g\|_{\mathscr{B}} \leqslant R\right\}$. Hypotheses $\left(\mathrm{H}_{+}\right)$and $\left(\mathrm{H}_{3}\right)$ imply that the continuity is uniform in $y$. So, there exists $\eta$ (depending on $R, \rho, n$ ) such that if $\|g\|_{\infty}<2 \eta$ and $\|g\|_{\mathscr{B}} \leqslant R$ then

$$
\sup _{y} \sup _{t_{i}^{n} \leqslant t \leqslant T}\left|\int_{[0, t] \cap \Delta_{i}^{n}} \sigma_{j}(t, s, y) \dot{g}_{s}^{j} \mathrm{~d} s\right| \leqslant \frac{\rho}{2 n} .
$$

Then, (4.14) and (4.15) imply

$$
\begin{aligned}
P\left(B_{i}\right) & \leqslant \sup _{y} \exp \left(-\frac{1}{\varepsilon^{2}} \inf \left\{\frac{1}{2} \int_{t_{i}}^{T}\left|\dot{g}_{s}\right|^{2} \mathrm{~d} s,\|g\|_{\infty} \leqslant 2 \eta, \sup _{t_{i} \leqslant t \leqslant T}\left|\int_{[0, t] \cap \Delta_{i}^{n}} \sigma_{j}(t, s, y) \dot{g}_{s}^{j} \mathrm{~d} s\right|>\frac{\rho}{2 n}\right)\right. \\
& \leqslant \exp \left(-\frac{R}{\varepsilon^{2}}\right),
\end{aligned}
$$

for a suitable $\eta$ (depending on $R, \rho$ and $n$ ). Finally, (4.12), (4.13) and (4.16) imply (4.11) for $\mu$ (depending on $R$ and $\rho$ ) small enough. This argument has been used in Baldi and SanzSole (1991) to show the equivalence between the estimate (1.3) and the large-deviation principle for the couple $\left\{\left(X_{1}^{\varepsilon}, X_{2}^{\varepsilon}\right), \varepsilon>0\right\}$.

Using the auxiliary results established so far it is now possible to prove Proposition 5.

Proof of Proposition 5. Set $A^{\varepsilon}=\left\{\left\|Y^{\varepsilon}-S(g)\right\|_{\infty}>\rho,\|\varepsilon W\|_{\infty}<\eta\right\}$. The inequality (4.6) yields for all $n \in \mathbb{N}, \mu>0, A^{\varepsilon} \subset \cup_{i=1}^{2} A_{i}^{\varepsilon, n, \mu}$, with

$$
\begin{gathered}
A_{1}^{\varepsilon, n, \mu}=\left\{\left\|Y^{\varepsilon}-Y^{\varepsilon, n}\right\|_{\infty}>\mu\right\}, \\
A_{2}^{\varepsilon, n, \mu}=\left\{\sup _{0 \leqslant t \leqslant T}\left|\int_{0}^{t} \varepsilon \sigma_{j}\left(t, s, Y_{s}^{\varepsilon, n}\right) \mathrm{d} W_{s}^{j}\right|>\frac{\rho}{\bar{K}_{1}},\|\varepsilon W\|_{\infty}<\eta,\left\|Y^{\varepsilon}-Y^{\varepsilon, n}\right\|_{\infty} \leqslant \mu\right\} .
\end{gathered}
$$

Fix $R, \rho>0$. Proposition 8 yields the existence of $\mu_{0}(R, \rho)>0$ and $\eta_{0}(R, \rho, n)>0$ (for each fixed $n \in \mathbb{N}$ ) such that for all $\mu \leqslant \mu_{0}, \eta \leqslant \eta_{0}$ and $\varepsilon \in(0,1]$,

$$
P\left(A_{2}^{\varepsilon, n, \mu}\right) \leqslant \exp \left(-\frac{R}{\varepsilon^{2}}\right) .
$$

By Proposition 7 , there exists $n_{0}\left(R, \mu_{0}\right) \in \mathbb{N}$ such that for any $n \geqslant n_{0}$ and $\varepsilon \in\left(0, \varepsilon_{0}\right]$

$$
P\left(A_{1}^{\varepsilon, n, \mu}\right) \leqslant \exp \left(-\frac{R}{\varepsilon^{2}}\right) .
$$


Then, (4.3) holds true with $\eta=\eta_{0}\left(R, \rho, n_{0}\right)$. This finishes the proof of Proposition 5 under $(\mathrm{H}) \vee\left(\mathrm{H}_{+}\right)$. Assume now the weaker hypothesis $(\mathrm{H})$. Fix $g \in \mathscr{H}$ and set

$$
\tau^{\varepsilon}(\rho)=\inf \left\{t>0: \sup _{0 \leqslant s \leqslant t}\left|Y_{s}^{\varepsilon}-S(g)_{s}\right|>\rho\right\} \wedge T .
$$

By definition,

$$
\sup _{t \leqslant \tau^{\varepsilon}(\rho)}\left|Y_{t}^{\varepsilon}\right| \leqslant \rho+\|S(g)\|_{\infty}:=C(\rho, g) .
$$

Consider the new coefficients

$$
\tilde{\sigma}(t, s, x)= \begin{cases}\sigma(t, s, x), & \text { if }|x| \leqslant C(\rho, g), \\ \sigma\left(t, s, \frac{x}{|x|} C(\rho, g)\right), & \text { if }|x|>C(\rho, g),\end{cases}
$$

and $\tilde{b}$ defined in an analogous way. Then, (4.17) and the local property of the stochastic integral yield

$$
\begin{aligned}
P\left\{\left\|Y^{\varepsilon}-S(g)\right\|_{\infty}>\rho,\|\varepsilon W\|_{\infty}<\eta\right\} & \leqslant P\left\{\sup _{t \leqslant \tau^{\varepsilon}(\rho)}\left|Y_{t}^{\varepsilon}-S(g)_{t}\right|>\rho,\|\varepsilon W\|_{\infty}<\eta\right\} \\
& =P\left\{\sup _{t \leqslant \tau^{\varepsilon}(\rho)}\left|\tilde{Y}_{t}^{\varepsilon}-\tilde{S}(g)_{t}\right|>\rho,\|\varepsilon W\|_{\infty}<\eta\right\},
\end{aligned}
$$

where $\left\{\tilde{Y}_{t}^{\varepsilon}, t \in[0, T]\right\}$ (or $\left\{\tilde{S}(g)_{t}, t \in[0, T]\right\}$ ) is defined as in (4.2) (or (2.1)) with coefficients $\tilde{\sigma}$ and $\tilde{b}$ which satisfy also assumptions $\left(\mathrm{H}_{+}\right)$. The first part of the proof allow us to conclude that Proposition 5 holds under $(\mathrm{H})$. Hence the LDP is checked.

\section{Applications}

In this section we discuss two different applications of Theorem 1.

\subsection{Stochastic differential equations driven by a fractional Brownian motion}

For any $H$ in $(0,1)$ the fractional Brownian motion of index $H,\left\{W_{t}^{H}, t \geqslant 0\right\}$ is the unique centred Gaussian process whose covariance kernel is given by

$$
R_{H}(s, t)=\frac{V_{H}}{2}\left(s^{2 H}+t^{2 H}-|t-s|^{2 H}\right),
$$

where $V_{H}$ is the normalizing constant

$$
V_{H}=\frac{\Gamma(2-2 H) \cos (\pi H)}{\pi H(1-2 H)} .
$$

It is known that the fractional Brownian motion admits the representation 


$$
W_{t}^{H}=\int_{0}^{t} K_{H}(t, s) \mathrm{d} W_{s},
$$

where $K_{H}(t, s)$ is the square root of the covariance operator:

$$
R_{H}(s, t)=\int_{0}^{1} K_{H}(s, r) K_{H}(t, r) \mathrm{d} r .
$$

More precisely,

$$
K_{H}(t, r)=\frac{(t-r)^{H-\frac{1}{2}}}{\Gamma\left(H+\frac{1}{2}\right)} F\left(\frac{1}{2}-H, H-\frac{1}{2}, H+\frac{1}{2}, 1-\frac{t}{r}\right) 1_{[0, t)}(r),
$$

where $F$ is the Gauss hypergeometric function. We refer to Decreusefond and Üstünel (1999) and the references therein for a detailed presentation of these notions.

Following the work of Coutin and Decreusefond (1997), by a stochastic differential equation driven by a fractional Brownian motion we mean an equation of the form

$$
X_{t}=x_{0}+\int_{0}^{t} K_{H}(t, s) \tilde{b}\left(s, X_{s}\right) \mathrm{d} s+\int_{0}^{t} K_{H}(t, s) \tilde{\sigma}\left(s, X_{s}\right) \mathrm{d} W_{s},
$$

where $\tilde{b}$ and $\tilde{\sigma}$ are deterministic functions and $\left\{W_{t} ; t \geqslant 0\right\}$ is the standard Brownian motion appearing in the representation (5.1).

Assume that $\tilde{\sigma}$ and $\tilde{b}$ are bounded measurable functions Lipschitz in $x$. Then, the coefficients

$$
\begin{gathered}
\sigma(t, s, x)=K_{H}(t, s) \tilde{\sigma}(s, x), \\
b(t, s, x)=K_{H}(t, s) \tilde{b}(s, x)
\end{gathered}
$$

satisfy assumptions $(H)$ in Theorem 1 provided $\frac{1}{2} \leqslant H<1$. As a consequence, from Theorem 1 we deduce an LDP for a family of processes obtained by small perturbations of equation (5.2).

\subsection{Hyperbolic stochastic partial differential equations}

Let $T=[0,1]^{2}$ and $\left\{W_{s, t},(s, t) \in T\right\}$ be a Wiener sheet. Consider the hyperbolic stochastic partial differential equation

$$
\frac{\partial^{2} X_{s, t}}{\partial s \partial t}=a_{3}\left(s, t, X_{s, t}\right) \dot{W}_{s, t}+a_{4}\left(s, t, X_{s, t}\right)+a_{1}(s, t) \frac{\partial X_{s, t}}{\partial s}+a_{2}(s, t) \frac{\partial X_{s, t}}{\partial t},
$$

with deterministic initial condition $X_{s, t}=x_{0}$ if $(s, t) \in T$, s.t $=0$.

We assume the following set of assumptions $(F)$ :

$\left(\mathrm{F}_{1}\right) a_{i}: T \rightarrow \mathbb{R}, i=1,2$ are bounded.

$\left(\mathrm{F}_{2}\right)$ There exists a constant $K>0$ such that for all $(s, t) \in T$ and $i=3,4$ 


$$
\begin{gathered}
\left|a_{i}(s, t, y)-a_{i}(s, t, x)\right| \leqslant K|x-y|, \\
\left|a_{i}(s, t, 0)\right| \leqslant K .
\end{gathered}
$$

A solution of (5.3) is a stochastic process $\left\{X_{z}, z \in T\right\}$ satisfying

$$
X_{z}=x_{0}+\int_{R_{z}} \gamma_{z}(\eta)\left\{a_{3}\left(\eta, X_{\eta}\right) \mathrm{d} W_{\eta}+a_{4}\left(\eta, X_{\eta}\right) \mathrm{d} \eta\right\},
$$

where $\eta=(u, v), z=(s, t), R_{z}$ denotes the rectangle $[0, s] \times[0, t]$ and $\gamma_{z}(\eta)$ is the Green function associated with the second-order differential operator

$$
\mathscr{T} f(s, t)=\frac{\partial^{2} f(s, t)}{\partial s \partial t}-a_{1}(s, t) \frac{\partial f(s, t)}{\partial s}-a_{2}(s, t) \frac{\partial f(s, t)}{\partial t} .
$$

Under assumptions $(F)$, the Green function is bounded and Lipschitz continuous in the variable $z$, uniformly in $\eta$, and we can prove the existence and uniqueness of a continuous and adapted process $\left\{X_{z}, z \in T\right\}$ bounded in $L^{p}$, for any $p \geqslant 2$.

Consider the family $\left\{X_{z}^{\varepsilon}, \varepsilon>0\right\}$ of processes that satisfy the equation

$$
X_{z}^{\varepsilon}=x_{0}+\int_{R_{z}} \gamma_{z}(\eta)\left\{\varepsilon a_{3}\left(\eta, X_{\eta}^{\varepsilon}\right) \mathrm{d} W_{\eta}+a_{4}\left(\eta, X_{\eta}^{\varepsilon}\right) \mathrm{d} \eta\right\}
$$

The coefficients

$$
\begin{gathered}
\sigma(z, \eta, x)=\gamma_{z}(\eta) a_{3}(\eta, x), \\
b(z, \eta, x)=\gamma_{z}(\eta) a_{4}(\eta, x)
\end{gathered}
$$

satisfy conditions analogous to assumptions $(\mathrm{H})$. Applying the same arguments as in the proof of Theorem 1 we can establish the following result:

Theorem 9. Assume (F). The family $\left\{X^{\varepsilon}, \varepsilon>0\right\}$ of solutions of (5.4) satisfies a largedeviation principle on the space $\mathscr{C}_{x_{0}}(T, \mathbb{R})$ (continuous functions which take the value $x_{0}$ in the axes) with rate function

$$
I(\varphi)=\inf \left\{\frac{1}{2} \int_{T}\left|\frac{\partial^{2} g}{\partial s \partial t}\right|^{2} \mathrm{~d} s \mathrm{~d} t, \varphi=S(g), g \in \mathscr{H}\right\},
$$

where

$$
S(g)(z)=x_{0}+\int_{R_{z}} \gamma_{z}(\eta)\left\{a_{3}(\eta, S(g)(\eta)) \frac{\partial^{2} g}{\partial u \partial v} \mathrm{~d} \eta+a_{4}(\eta, S(g)(\eta)) \mathrm{d} \eta\right\},
$$

and $\mathscr{H}$ is the set of absolutely continuous functions $g \in \mathscr{C}_{0}(T, \mathbb{R})$ such that $\int_{T}\left|\partial^{2} g / \partial s \partial t\right|^{2} \mathrm{~d} s \mathrm{~d} t<\infty$.

This result is obtained in Rovira and Sanz-Solé (1997) assuming that $a_{i}, i=3,4$, are Lipschitz functions in all the variables, the derivatives $\partial a_{3} / \partial s, \partial a_{3} / \partial t$ and $\partial^{2} a_{3} / \partial t \partial s$ exist and are Lipschtiz functions, and $a_{i}, i=1,2$, are differentiable with bounded derivatives. 


\section{Acknowledgements}

This work was supported by Dirección General de Investigación Científica y Técnica grants PB96-0087 (to David Nualart) and PB96-0088 (to Carles Rovira).

\section{References}

Azencott, R. (1980) Grandes déviations et applications. In P. L. Hennequin (ed.), École d'Été de Probabilités de Saint-Flour VIII, 1978, Lecture Notes in Math. 774, pp. 1-176. Berlin: SpringerVerlag.

Baldi, P. and Sanz-Solé, M. (1991) Une remarque sur la théorie des grandes déviations. In J. Azéma, P.A. Meyer and M. Yor (eds), Séminaire de Probabilités XXV. Lecture Notes in Math. 1485, pp. 345-348. Berlin: Springer-Verlag.

Coutin, L. and Decreusefond, L. (1997) Stochastic differential equations driven by a fractional Brownian motion. Preprint.

Decreusefond, L. and Üstünel, A.S. (1999) Stochastic analysis of the fractional Brownian motion. Potential Anal., 10, 177-214.

Doss, H. and Priouret, P. (1983) Petites perturbations des systèmes dynamiques avec reflexion. In J. Azéma and M. Yor (eds), Séminaire de Probabilités XVII, 1981/82, Lecture Notes in Math. 986, pp. 353-370. Berlin: Springer-Verlag.

Ledoux, M. (1990) A note on large-deviations for Wiener chaos. In J. Azéma, P.A. Meyer and M. Yor (eds), Séminaire de Probabilités XXIV, 1988/89, Lecture Notes in Math. 1426, pp. 1-14, Berlin: Springer-Verlag.

Mayer-Wolf, E., Nualart, D. and Pérez-Abreu, V. (1992) Large deviations for multiple Wiener-Itô integral processes. In J. Azéma, P.A. Meyer and M. Yor (eds), Séminaire de Probabilités XXVI, Lecture Notes in Math. 1526, pp. 11-31. Berlin: Springer-Verlag.

Millet, A., Nualart, D. and Sanz-Solé, M. (1992) Large deviations for a class of anticipating stochastic differential equations. Ann. Probab., 20, 1902-1931.

Mueller, C. (1991) Limit results for two stochastic partial differential equations. Stochastics Stochastics Rep., 37, 175-199.

Priouret, P. (1982) Remarques sur les petites perturbations de systèmes dynamiques. In J. Azéma and M. Yor (eds), Séminaire de Probabilités XVI, 1980/81, Lecture Notes in Math. 921, pp. 184-200. Berlin: Springer-Verlag.

Rovira, C. and Sanz-Solé, M. (1996) The law of the solution to a nonlinear hyperbolic SPDE. J. Theoret. Probab., 9, 863-901.

Rovira, C. and Sanz-Solé, M. (1997) Large deviations for stochastic Volterra equations in the plane. Potential Anal. To appear.

Sowers, R.B. (1992) Large deviations for a reaction-diffusion equation with non-Gaussian perturbations. Ann. Probab., 20, 504-537.

Ventzell, A.D. and Freidlin, M.I. (1970) On small random perturbations of dynamical systems. Russian Math. Surveys, 25, 1-55.

Walsh, J.B. (1986) An introduction to stochastic partial differential equations. In P.L. Hennequin (ed.), École d'Été de Probabilités de Saint-Flour XIV, 1984, Lecture Notes in Math. 1180, pp. 266473. Berlin: Springer-Verlag.

Received January 1998 and revised March 1999 